\title{
Performance Analysis of AODV, CBRP, DSDV and DSR MANET Routing Protocol using NS2 Simulation
}

\author{
Awadhesh Kumar ${ }^{1}$, Prabhat Singh ${ }^{2}$, Vinay Kumar ${ }^{3}$, Dr. Neeraj Tyagi ${ }^{4}$ \\ ${ }^{1,2,3}$ Department of Computer Science and Engineering, Kamla Nehru Institute of technology, Sultanpur, India \\ ${ }^{4}$ Department of Computer Science and Engineering, MNNIT, Allahabad, India \\ awadhesh@knit.ac.in, prabhatsingh@gmail.com, vinayknit@hotmail.com,neerajtyagi@mnnit.ac.in
}

\begin{abstract}
A Mobile Ad-hoc Network (MANET) is a collection of multi-hop wireless mobile nodes among which the communication is carried out without any centralized control or fixed infrastructure. MANET is a self-organized, self-configurable network having no infrastructure, and in which the mobile nodes move arbitrarily. The wireless link in the network are highly error prone and can go down frequently due to the mobility of nodes, interference and less infrastructure. Hence, because of the highly dynamic environment routing in MANET is a very difficult task. Over the last decade various routing protocols have been proposed for the mobile ad-hoc network and the most important among all of them are AODV, DSR, DSDV and CBRP. This research paper gives the overview of these routing protocols as well as the characteristics and functionality of these routing protocols along with their pros and cons and then make their comparative analysis in order to measure the performance of the network. The main objective of this paper is to compare the performance of all the four routing protocols and then to make the observations about how the performance of these routing protocols can be improved. Performance of these routing protocols are compared on the basis of various parameters such as throughput, delay and packet delivery ratio.
\end{abstract}

Index Terms - Ad Hoc On Demand Distance Vector (AODV), Cluster Based Routing Protocol (CBRP), Destination-Sequenced Distance Vector (DSDV), Dynamic Source Routing (DSR).

\section{INTRODUCTION}

Over the last decade, researchers have made various researches in the field of mobile computing especially MANETs. A Mobile ad-hoc network (MANETs) is a self-organized, arbitrarily developed network and can easily adopt in working environment. Basically, MANET is the collection of wireless mobile nodes that can interact and communicate with each other, without having the centralized and established infrastructure. MANETs have converted the dream of getting connected "anywhere and at any time" in to the reality. MANETs are useful in various application areas such as: communication in the battlefields, institutions and colleges, military areas, disaster recovery areas, law and order maintenance, traffic control areas, medical field, conferences and convocations etc. In MANET, all the nodes are mobile nodes and their topology changes rapidly. The Internet Engineering Task Force (IETF) created a MANET working group to deal with the challenges faced during the construction of the MANET routing protocols. These protocols are basically classified in to three basic types such as: reactive (on demand), proactive (table-driven) and hybrid. One of the basic goals of the mobile ad-hoc network is to establish correct and efficient route between the mobile nodes so that communication between the sender and receiver is effective.

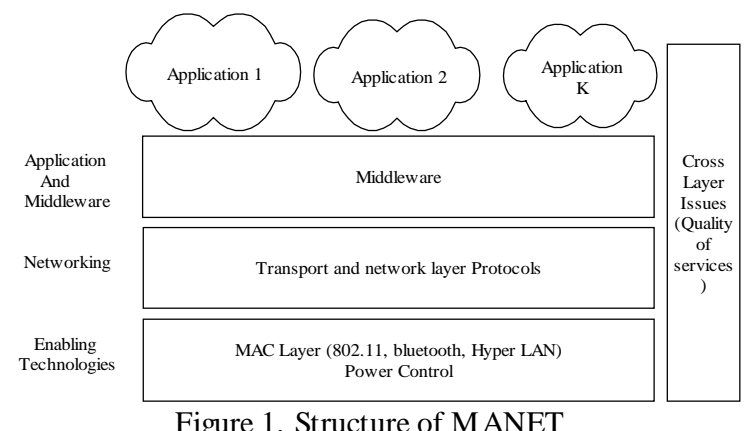

In Proactive (table driven) routing protocols, each node maintain one or more routing table which contain information about every other node in a network. Routing tables are updated by all the nodes in order to maintain a consistent and up to date view of the network. In table driven routing protocol, continuous broadcasting of messages is done in order to establish routes and maintain them. One of the basic advantages of proactive routing protocol is that route from source to destination is easily available without any overhead, as they are independent of traffic profiles. Various proactive routing protocols are: DSDV [1], [2], DBF [3], GSR [4], WRP [5] and ZRP [6]. 
In reactive (on demand) routing protocol, creation of routes is done when it is required. When some packets are to be send from source to destination, it may invoke the route discovery mechanism to find the path to the destination. The route is valid, till the destination is reached or it is no longer be required in the future. Some of the reactive routing protocols are: DSR [1] [3], AODV [8] [6] and TORA [2].

In hybrid protocol routing, we combine the benefits of both the reactive as well as proactive. Hybrid protocols are basically dependent on the network size for their functionality. The remaining portion of this paper is categorized as follows. In section 2, we will discuss the various issues and challenges faced by the MANET. In section 3, we will focus on the routing protocols such as AODV, DSR, DSDV, and CBRP. We will compare the reactive routing protocols with proactive routing protocols and understands the difference between them that affect the performance of these routing protocols. In section 4, we present the simulation of these routing protocols and compare their performance. In section 5, we analyze various results that are obtained from the simulations. Finally, we conclude the paper in section 6 .

\section{CHA LLENGES FA CED BY THE MANETS}

MANETs are very much different from the wireless network infrastructures. MANETs has to face various challenges in order to achieve best Quality of Service for the underlying network. Some of these challenges are as follows:

1. Unicast and multicast routing.

2. Topology changes dynamically.

3. Speed and network overhead.

4. Limited power supply and bandwidth.

5. Quality of service and secure routing.

6. Scalable and energy efficient routing.

These challenges are faced at the different layers of MANETs shown in the figure 2. It represents the layered architecture of the OSI model and entitled the challenges of MANETs regarding these layers.

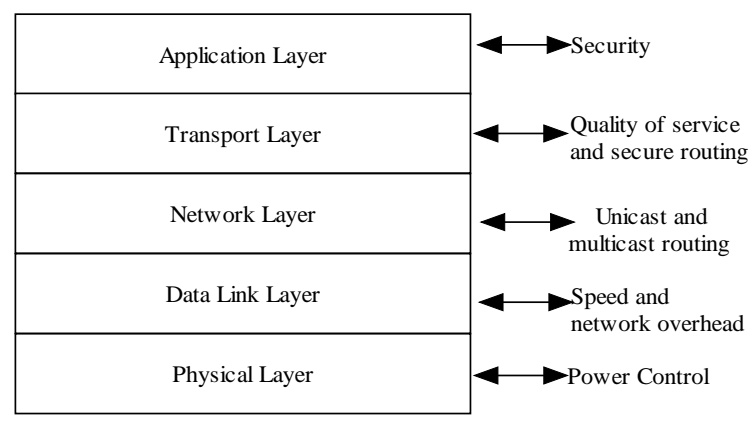

Figure 2. Challenges are faced at the different layers of MANETs

\section{ROUTING PROTOCOLS}

\section{A. Ad Hoc On Demand Distance Vector (AODV)}

Basically AODV is the improvement of DSDV routing algorithm. It is collectively based on the combination of both DSDV and DSR. The main aim of AODV routing algorith $m$ is to provide reliable and secure data transmission over the MANETs. In this routing protocol, route maintenance from one node to every other node is not considered in the network. Whereas in AODV, route are discovered only when they are needed as well as they are maintained only as long as they are required. The key steps used in the AODV algorithm are defined below:

\section{Route creation-}

Route creation process is initiated when the node wants to send the data packet to the destination node but does not find the valid route to send the packet. This process is initiated as such: when a particular node want to send the data packet to the destination node then, the routing table entries are checked in order to verify whether there exists a route between the source and destination or not. If the route exists, then the packet is forwarded to the next hop towards the destination. When the route does not exists, and then the route discovery process is initiated. AODV starts the route discovery process using the route request (RREQ) and route reply (RREP). The source node will create the route request packet(RREQ) containing its sequence number, its IP address, destination node sequence number, destination IP address and broadcasting ID. Whenever the source node initiates the RREQ, the broadcast ID is incremented each time. As the sequence number is used to identify the timeliness of each data packet and broadcast ID and the IP address identify the unique identifier for route request so that they can uniquely identify each of the request. The data packet sends the request using the RREQ message and gets back the reply in the form of RREP message if the route is discovered between the nodes. The source node sends the RREQ packet to its neighbors as well as set the timer in order to wait for the RREP message. In order to describe RREQ, reverse route entry is set up by the node in its routing table. This enables us to know how we can forward the route reply (RREP) to the source. Moreover, a time period is associated with the reverse route entry and if this route entry is not used within the given time period then only the route information is deleted. If in any case RREQ is lost during the transfer of packet then the source node again initiates route discovery mechanis $\mathrm{m}$. 


\section{Route maintenance-}

Route that has been created by the source node and destination node is being maintained as long as it is needed by the source node. Since nodes in the MANETs are mobile and if the source node is in the mobile state during the active session, it again restarts the route discovery mechanis $\mathrm{m}$ in order to establish new routes from source to destination.

Otherwise, if some of the intermediate node or the destination node is in the mobile state during the active session, then the nodes initiates the RERR message that affects the above neighbors and the nodes. As a result of which these nodes forward the RERR message to the predecessor nodes and can be continued until the source node is reached. When RERR is received by the source node, then the node stop sending the data or it can again start the route discovery mechanis mby sending the RREQ message.

\section{Advantages-}

- Loop free routing.

- Optional multicast.

- $\quad$ Reduced control overhead. Disadvantages-

- Bi-directional connection needed in order to detect a unidirectional lin $\mathrm{k}$.

- Delay caused by the route discovery process.

\section{B. Dynamic Source Routing (DSR)-}

DSR is the reactive routing protocol which is able to manage MANETs without using the periodic table update messages like proactive routing protocols does. Specially, DSR are designed to make use in multi-hop wireless ad-hoc networks. Ad-hoc network enables the network to be self-organizing and selfconfiguring which means that there is no need of existing network infrastructure. This protocol basically focuses on the two different phases i.e. route discovery and route maintenance. In route discovery phase, source node sends the packet to the destination node. In route maintenance phase, protocol detects when the topology of the network has changed and decides if an alternative route has been used or the route discovery protocol must be started to find the new path. Route discovery and route maintenance phase only give responses when they receive the request.

\section{Advantages-}

- $\quad$ DSR does not need the routing table for making the periodic updates.
- Intermediate nodes are able to utilize the route cache information efficiently to reduce the control overhead.

- Bandwidth saving because it does not require any HELLO messages.

\section{Disadvantages-}

- Route maintenance protocol does not locally repair the broken link.

- This protocol is only been efficient for less than 200 nodes in MANETs.

- There is small time delay if the beginning of the new connection takes place.

\section{Destination- Sequenced Distance Vector Routing-}

The DSDV algorithm is basically the amendments made in distributed bellman ford algorithm, which provides loop free routes. It gives us the single path from source to destination using distance vector routing protocol. In order to reduce the amount of overhead in a network two types of updates packets are transferred i.e. full dump and incremental packet. Full dump broadcasting carry all the routing information while the incremental dump broadcasting will carry information that has changed since last full dump irrespective of the two types, broadcasting is done in the network protocol data unit(NPDU). Full dump requires multiple NPDU's whereas incremental dump requires one NPDU's to fit in all the information. Incremental update packets are sent more easily and frequently than the full dump packets. DSDV introduces the large amount of overhead to the network due to the requirement of periodic update messages. Hence, this protocol is not suitable for the large network because large portion of the network bandwidth is used for the updating of messages.

1. Management of the routing table-

The routing table for each and every node consists of a list of all available nodes, their next hop to the destination, their metric and a sequence number generated by the destination node. With the help of the MANETs, routing table is used to send the data packets. Routing table can be kept consistent with the dynamically changing topology of ad-hoc network by periodically updating the routing table with some small changes in the network. Hence, mobile nodes provide their routing information by broadcasting the routing table update packet. The metric of the update packet starts with the initial value of one for one hop 
neighbors and goes on incremented with each forwarding node. The receiving node updates their routing tables if the sequence number of the update is greater than the current node or equal to the current node. Fluctuations in the routing table are minimized by delaying the advertisement of routes until we find the best route.

2. Changes in the topology-

DSDV responds to the broken links by authorizing all the routes that contain this link. The routes are immediately assigned a metric as well as the incremented sequence number. Physical and data link layer components are used to detect the broken links or if the node does not receive broadcast packets from its neighbors node. Then, immediately the detecting node will broadcast an update packet and inform all the other nodes about the broadcasting mechanism. Route will again be reestablished when the routing table is broadcasted by the node.

Advantages-

- Guarantees loop free path.

- Count to infinity problem is reduced in DSDV.

- With incremental updates, we can avoid extra traffic.

- DSDV maintain the best possible path instead of maintaining the multiple paths to the destination this reduces the amount of space in the routing table.

\section{Disadvantages-}

- It does not support the multipath routing.

- Difficult to determine the delay for the advertisement of routes.

- Unnecessary advertising of routing information can result in the wastage of bandwidth.

D. Cluster Based Routing Protocol (CBRP)-

It is a reactive and on demand routing protocol in which nodes are divided in to the clusters. This protocol uses the clustering structure for the routing procedures. Clustering is defined as the phenomenon that divides the network in to the interconnected sub networks. Each cluster has the cluster head which acts as the coordinator in sub networks. Each cluster head has the base station within its cluster so that it can commun icate with other cluster heads. This is the reactive routing protocol which is to be used in MANETs. The protocol divides the nodes in to the number of clusters in a distributed manner. Each cluster contains the cluster head which contain the cluster information. Four possible states for the nodes are as follows: CLUSTERHEAD, GATEWAY, NORMAL and GATEWAY. Initially all the nodes are in the isolated state. Each node maintain the NEIGHBOR table, wherein the information about the other neighbor nodes are stored whereas, cluster heads maintain another table wherein the information about the other neighbor cluster head is stored [13].

\section{SIMULAT ION}

The main goal of the simulation is to analyze the performance of different MANETs routing protocol. The simulation is being performed by the NS2 simulator. It is the software that provides the simulations of wireless networks and open source software. In our simu lation, we consider a network of 5 nodes that are placed randomly within a $360000 \mathrm{~m}^{2}$ areas and operating over 300 seconds. In this, multip le run with different node speed and number of nodes are calculated for each scenarios and collected data is averaged further in order to understand the performance of different routing protocols. For calculating the performance of different routing protocol, we require both qualitative as well as quantitative metrics. So me of the quantitative metrics are used to compare the performance of different routing protocols which are as follows:

A. Throughput- It basically defines as ratio of the number of packets delivered to the total number of packets sent.

B. Packet delivery ratio- It is the ratio of total number of packets successfully delivered to the destination nodes to the total number of packets send by the source nodes. It basically describes the percentage of packets that reach the destination.

C. Minimum and maximum delay- Minimum delay is the minimum time taken by the packets to reach the next node and maximum delay is maximum time taken by the packets to reach the next node.

D. Average End to End delay- It is the total time taken by all the packets to reach the destination.

E. Network parameters- In this, simulation time is the time taken during the simulation run that is time between the starting of simulation and end 
of the simulation. Network size basically determines the number of the nodes used and the area occupied by the network. The number of nodes used in this simulation is 5 .

\section{SIMULATION RESULTS AND DISCUSSIONS}

As seen in the figure-3, if the mobility increases AODV gives the maximum throughput, DSDV, DSR and CBRP throughput is decreased with the increase in node speed.

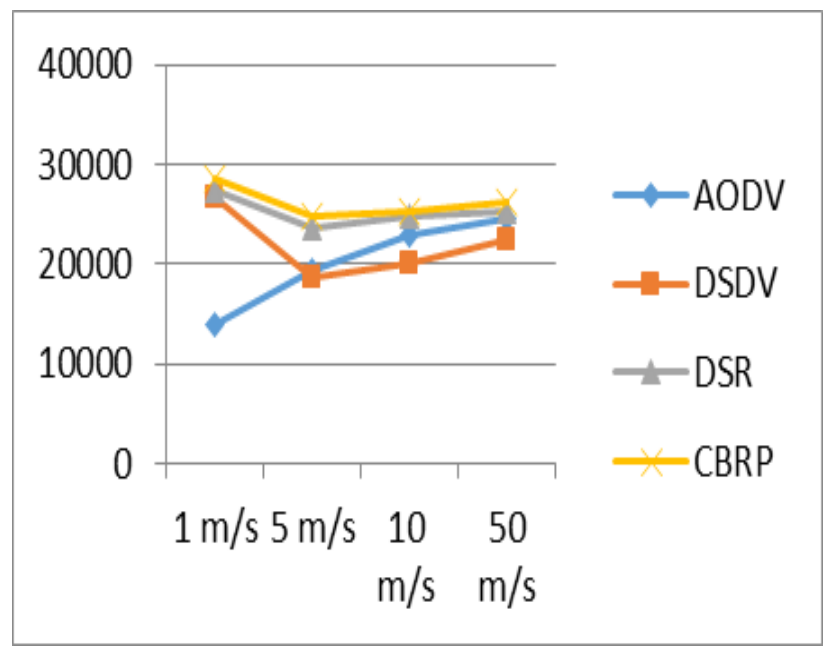

Figure 3. Average Throughput vs Speed

In figure-4 if node speed increases, average delay of AODV decreases while in DSDV, DSR and CBRP average delay increases with the increase in the node speed. It means packet delivery takes the less time as the node speed increases in AODV.

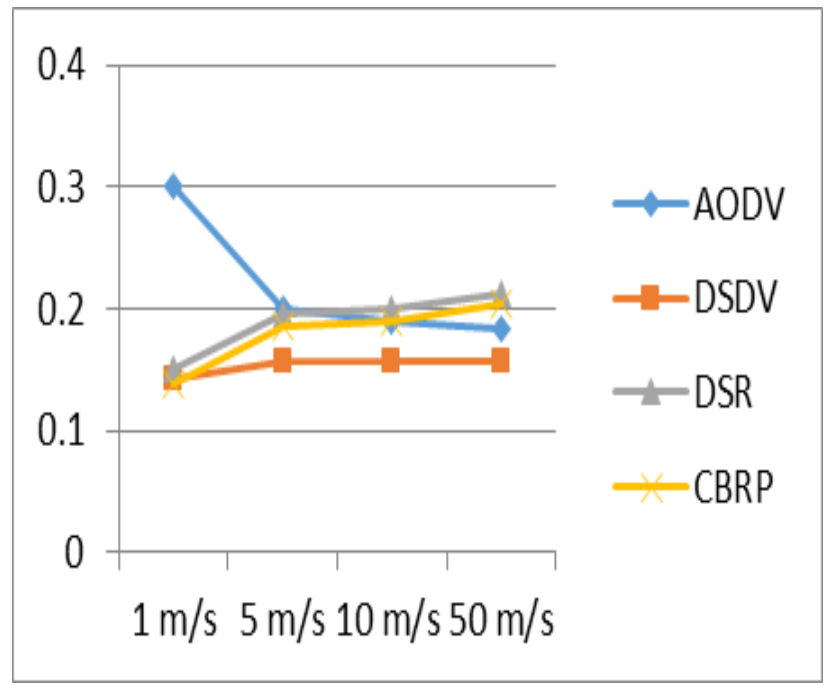

Figure 4. Average Delay vs Speed

In figure-5, as the mobility increases, the packet delivery ratio of AODV and DSDV decreases while it increases in DSR and CBRP. Hence in terms of packet delivery ratio, CBRP is better among all the four routing protocols.

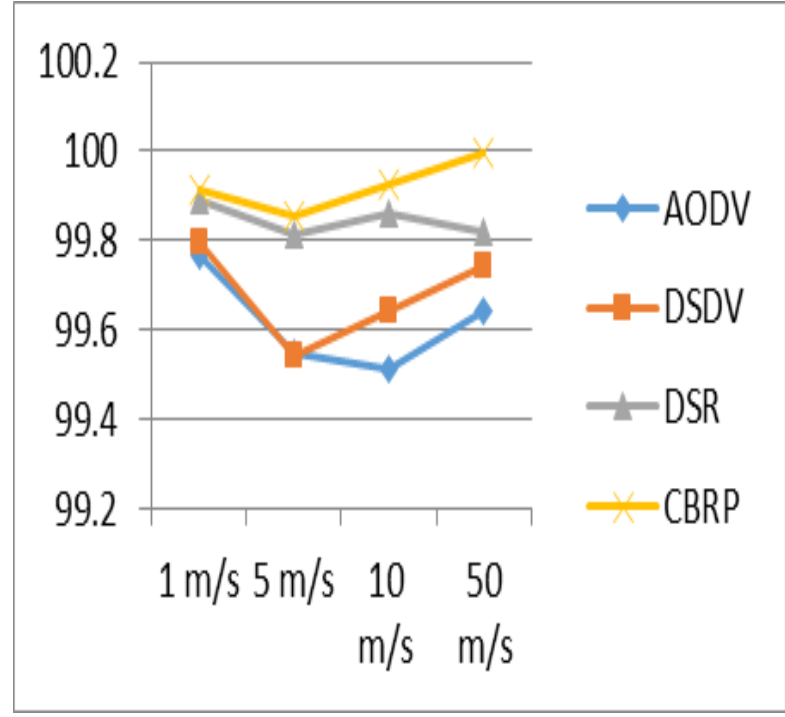

Figure 5. Packet Delivery Ratio vs Speed

\section{CONCLUSIONS}

This paper provides the detailed study of various routing protocols which are proposed for the mobile adhoc networks and also provide the classification of these routing protocols on the basis of their routing strategy. In this paper we have presented AODV, CBRP, DSR and DSDV and study their features, differences and characteristics. The performance of these routing protocols is analyzed with NS2 simulator with scenario of 5 nodes. The observations are made with variation in network mobility. After various analysis of network in different situations, we comes at the conclusion that AODV performs better than DSR, DSDV, and CBRP in terms of throughput and average delay while CBRP is proved to be better in terms of packet delivery ratio. All in all, by analyzing all the parameters, we come at the conclusion that the AODV routing protocol is better.

\section{REFERENCES}

[1]. David B. Johnson and David A. Maltz. Dynamic source routing in ad hoc wireless networks. Technical report, Carnegie Mellon University, 1996.

[2]. Mehran Abolhasan, Tadeusz Wysocki, and Eryk Dutkiewicz. A review of routingprotocols for mobile ad hoc networks. Technical report, Telecommunication and Information Research Institute, University of Wollongong, Wollongong, NSW 2522; Motorola Australia Research Centre, 12 Lord St., Botany, NSW 2525, Australia, 2003.

[3]. Xiaoyan Hong, Kaixin Xu, and Mario Gerla. Scalable routing protocols for mobile ad hoc networks. 2002.

[4]. Integration of mobile ad-hoc networks, EU project DAIDALOS, Susana Sargento, Institute of Telecommunications.

[5]. Mobile Ad Hoc Networking: An Essential Technology for Pervasive Computing Jun-Zhao Sun 
MediaTeam, Machine Vision and Media Processing Unit.

[6]. C. Siva Ram Murthy and B. S. Manoj, "Ad Hoc Wireless Networks, Architectures and Protocols", Second Edition, Low price Edition, Pearson Education, 2007.

[7]. International Journal of Computer Science \& Engineering Survey (IJCSES) Vol.1, No.1, August 2010 “ANALYZING THE MANET VARIATIONS, CHALLENGES, CAPACITY AND PROTOCOL ISSUES” G. S. Mamatha1 and Dr. S. C. Sharma

[8]. Jochen Schiller. Mobile Communications. AddisonWesley, 2000.

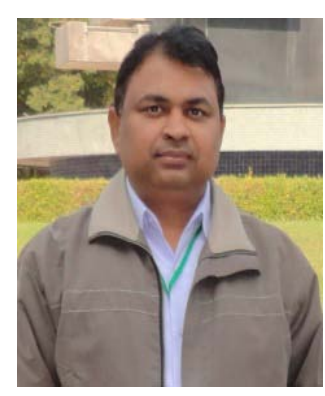

Awadhesh Kumar, graduated (B.E.) in 1999 from G.B. Pant Engineering, Puari (Garhwal) and completed his Post Graduation (M.Tech.) from Gautam Buddh Technical University, Lucknow U.P. and pursuing his research from MNNIT, Allahabad. Presently he is working as an Associate Professor in the Computer Sc.\& Engg. Dept., Kamla Nehru Institute of Technology, Sultanpur U.P. .

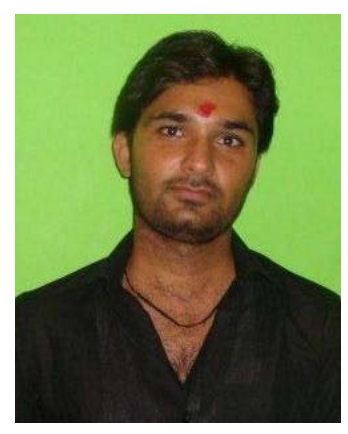

Prabaht Singh graduated from Priyadarshini College of Computer Sciences, greater noida, Uttar Pradesh in Computer Science \& Engineering in 2010. He is pursuing M.Tech in the department of Computer Science \& Engineering, Kamla Nehru Institute of Technology, Sultanpur (Uttar Pradesh).

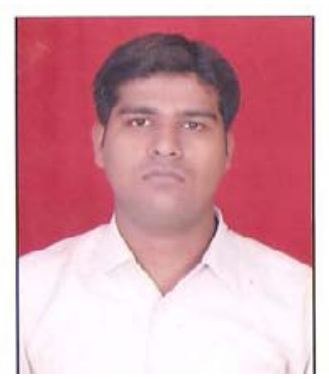

Vinay Kumar graduated Uttar Pradesh Technical University, in Computer Science \& Engineering in 2010. He is pursuing M.Tech in the department of Computer Science \& Engineering, Kamla Nehru Institute of Technology, Sultanpur (Uttar Pradesh).

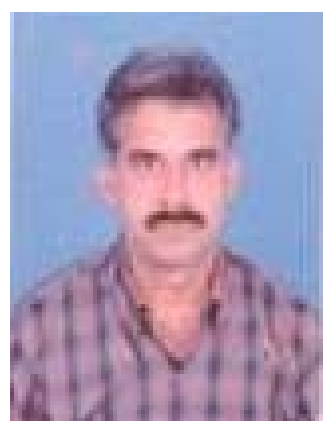

Dr. Neeraj Tyagi is working as Professor in the department of Computer Science \& Engineering, MNNIT, Allahabad(Uttar Pradesh), India. His teaching and research interests including Computer Networks, Mobile Ad-Hoc Networks, wireless Networks and Operating Systems. He joined the department of Computer Science \& Engineering in 1989 at MNNIT, Allahabad and he has Also worked in Warman International- Australia, G.E (Capital) - U.S.A, Electronic Data Systems- U.S.A during 1999-2001. 